\title{
On the effect of long-term electrical stimulation on three-dimensional cell cultures: Hen embryo brain spheroids
}

\author{
Ivan S Uroukov' \\ Larry Bull² \\ 'Faculty of Applied Sciences; ${ }^{2}$ Faculty \\ of Computing, Engineering, and \\ Mathematical Sciences, University \\ of the West of England, Bristol, UK
}

\begin{abstract}
A comprehensive dataset of multielectrode array recordings was collected from three-dimensional hen embryo brain cell cultures, termed spheroids, under long-term electrical stimulation. The aim is to understand the ongoing changes in the spiking activity under electrical stimulation within the lifetime of 14-72DIV of the neuronal networks contained therein. The spiking dynamics were analyzed and behavioral characteristics derived. Some effects on spiking patterns and exhaustion were followed in culture lifetime. With respect to the culture development, two main types of spiking exhaustion were found: one which materializes in the form of a drop in the sporadic (tonic) spiking frequency at the later maturation stages; and another associated with decreasing spiking train appearance throughout an experimental period.
\end{abstract}

Keywords: brain, 3D cell culture, spheroids, long-term electrical stimulation, exhaustion, learning, multielectrode array (MEA), neuronal networks, spike generation

\section{Introduction}

The first simultaneous intra- and extracellular recordings from cultures of dissociated neurons through the use of multielectrode array (MEA) technology (Thomas et al 1972; Gross 1979; Heuschkel et al 2002) were reported by Pine (1980). Since then, MEAs have become an essential tool for the testing of the electrical response (Wagenaar et al 2004) at the network level to stimulation in a wide range of areas, including computation (Demarse et al 2001), pharmaceutical development (Morefield et al 2000; Stett et al 2003), and image processing (Ruaro et al 2005). The majority of this work utilises dispersed monolayer cultures but Wagenaar and colleagues (2006) have recently suggested denser monolayer cultures should be used. However, it has long been known that aggregated neuronal cell cultures exhibit properties that are similar to their in vivo counterparts. Well established studies (DeLong 1970) showed identical structures to hippocampal architecture. Seeds (1971) showed close similarity of the temporal biochemical differentiation of brain cell aggregates to that seen during development in mice, which was much more so than equivalent monolayer cultures.

In the early 1990's, the three-dimensional (3D) brain cell culture model (based on murine and human foetal brain cells) was presented by Goldsmith and Berens (1990), called rotational-mediated cultures (RMA's). The RMA were found to contain diverse cell types including neurons, astrocytes, oligodendrocytes, epedymal cells, macrophages, endothelial cells, and fibroblasts. "These components may generate an extracellular matrix which mimics the in vivo mileu and there fore the intact brain itself' (Goldsmith and Berens 1990; p. 236). Goldsmith and Berens (1990) show only GFAP staining for 13 days in vitro (DIV) RMA where a glial structure of micro vials and protrusions are presented in the outer layer area in a network-like formation. Recently, a hen embryo brain aggregate model was presented as a suitable paradigm in which to perform in 
vitro toxicology studies relevant to organophosphate-induced neurotoxicity by Sales and colleagues (2004). We recently undertook the first MEA measurements of 3D hen embryo brain cell cultures (Uroukov et al 2006), finding complex spiking activity development during maturation. The spiking frequency ratio, that is, the ratio of stimulated to spontaneous frequency, was found to ramp up to around two by the end of the second week in vitro. Some in vivo studies are controversial, concerning the long term electrical stimulation, where possible morphological changes were linked to the stimulus charge (Babb et al 1977), or no consistent differences in the tissue were found and related to electrical stimulation where those levels were sufficient to elicit cortical evoked potentials (Güven et al 2005). We are particularly interested in the computational behavior of such aggregate cultures (Bull and Uroukov 2007), eg, capacity to exhibit memory and learning through induced plasticity. Long-term stimulation recordings are required in order to undertake such studies (eg, Shahaf and Marom 2001) and herein we describe a long-term study on the effect of electrical stimulation (Jimbo et al 1999) in order to begin exploring the spiking behavior under stimulation over time.

We have studied the bursting activity in the time course of the spheroids' lifetime and have observed various stages of spiking in a similar manner as Wagenaar and colleagues (2006) but we were able to extend their month-long monitoring to two and a half months. The effects of stimulation, spiking dynamics and adaptation (Eytan et al 2003) were traced in time and are described here. Distinctive train patterns were observed at specific stages in development, including spiking exhaustion. No comprehensive knowledge exists about spiking exhaustion and its reasons which impact upon the spiking activity for an inhibitory (Shu et al 2003) or excitatory response (Darbon et al 2002). In the present work, we study the change in spiking activity under long term stimulation without tetanizing (Jimbo et al 1998) the cultures. We were able to map two classes of exhaustion where they were recognized and analyzed over the lifetime scale of the cultures, one associated with the train activity and another with the sporadic activity. We also present GFAP/NF staining of thin sections of spheroids, showing a neuronal distribution in the inner side of the section.

\section{Materials and methods}

\section{Cell culturing}

Spheroid culturing is described in our previous publication (Uroukov et al 2006). Briefly, fertilized eggs type Galus Domesticus (Red Island Hens) were incubated for 7 days at $37^{\circ} \mathrm{C}$ in an egg incubator (Octagon 100; Brinsea Ltd., UK). On the day of spheroid preparation, eggs were removed from the incubator at a precise time in order that the E7 embryonic stage (Hamburger and Hamilton 1951) was reached. The neuroepithelial tissue was collected, cleaned and triturated to prepare a cell suspension that was then filtered through a $35 \mu \mathrm{m}$ Nybolt membrane. The plating cell concentration was $0.5 \times 10^{6}$ viable cells per $\mathrm{ml}$ of cell culture medium, $3 \mathrm{ml}$ per well in a six-well culture plate. Typically, a batch of 12 eggs is enough to culture up to 10 six-well plates. The six-well plates were placed on a gyrotatory shaker (Innova 2000; New Brunswick Scientific Co., Inc., UK) and cultured at $37{ }^{\circ} \mathrm{C}, 5 \% \mathrm{CO}_{2} / 95 \%$ air at $75 \mathrm{rpm}$. After $12 \mathrm{DIV}$ several thousands of spheroids per culture batch are ready to be used for sample preparation. Cell culture medium replacement took place every other day by removing some $50 \%$ of the old medium and refilling with fresh medium.

\section{Samples and cell cultures}

To produce a cell culture sample, to be assembled over 3D multi-electrode arrays, a selection procedure of the spheroids under a microscope is followed, eg, by size. The results reported here were obtained from twenty cultures.

\section{Sample preparation}

Multielectrode arrays (MCS-2100, 3D Multi Electrode Array, Multi Channel Systems MCS GmbH, Aspenhaustrasse 21, 72770 Reutlingen, Germany) with (3D-40 × $40 \times 70 \mu \mathrm{m}$, spaced on $200 \mu \mathrm{m}$ ) pyramid-shaped electrodes were used to record the electrical activity of the spheroids. To facilitate tissue adhesion to the MEA surface, a modification took place with $10 \mu \mathrm{g} / \mathrm{ml}$ aqueous solution of polymer ethylene imine (PEI) (Fluka Chemie AG, Buchs, Switzerland) under sterile circumstances. The selected volume of spheroids was dropped onto the centre of the MEA and rearranged over the electrodes. The MEAs were then refilled with culture medium and left to settle for 24 hours before experimentation.

\section{Recording}

The electrical recordings from the spheroids was performed with a 60 channel data acquisition system, where the sampling frequency of each channel was set to $25 \mathrm{kHz}$ and the single channel amplification kept at 1200 with a digital resolution of 12 bits. At these conditions, data sampling of the input band of spikes within $5 \mathrm{kHz}$ including a high pass $300 \mathrm{~Hz}$ filter was performed in a way that was similar to other studies (Guillory and Norman 1999; Jimbo et al 2000; Wagenaar et al 2004; Uroukov et al 2006). The spikes were detected by threshold 
depending upon the standard deviation and the offset of noise. A set of data was monitored and raw signal, filtered signal and spikes were chosen in order to perform fast and reliable recordings and analysis with the MC Rack software (Multi Channel Systems MCS, GmbH). The recorded data was written in the custom '*.med' MC Rack format and stored for further data analysis. The recorded signals were analyzed and the spike parameters extracted using the MC Rack software and analyzed with bespoke software as a post processing step. On a given MEA, a single spheroid was examined for their spiking response to the stimulus over time using one of the electrodes it covers. Note one spheroid contains an isolated 3D neuronal network (Uroukov et al 2006), which is potentially markedly different in many respects to monolayer cultures.

\section{Stimulation}

The stimulation of the spheroids was performed with the 8 channel programmed generator STG2008 with MC_Stimulus II software (Multi Channel Systems MCS, GmbH). An illustration of the experimental protocol is presented in Figure 1. The recordings and stimulation were performed in a repetitive manner as illustrated. Following Uroukov and colleagues (2006), a reference cycle of 10 minutes, denoted as $\mathrm{N}$, was recorded without any stimulation to ascertain the spheroid status before the repetitive stimulating. Recordings then consisted of multiple sets of 10 minute stimulation cycles (A to $\mathrm{G}$ ), with 5 minute rests in between. The stimulation signal consisted of a single sharp biphasic impulse of $300 \mu \mathrm{s}$ and voltages between $\pm 300 \mathrm{mV}$ each phase per sweep of $1 \mathrm{sec}$. This protocol was based upon that used in (Shahaf and Marom 2001).

\section{Spiking definition compatibility}

Since these types of cultures are not well-studied, indeed, we are pioneering their use with MEAs, there is not much to be shared from common MEA study terminology. We came to our definitions by comparing with the work of Wagenaar and colleagues (2006). We consider the following compatible from their study:

- - 'CB-Continuous Burst' or 'Sporadic activity' complies with 'Tonic Firing'

- -'MBI-Massive Burst Intervals' complies with 'Short Superburst'; length 100-300 $\mu \mathrm{sec}$

- -'MDB-Massive Dense Burst' complies with 'Long Superburst'; length 200-600 $\mu \mathrm{sec}$

- - 'MDBI- Massive Dense Burst Intervals' partly complies with 'Regular Superburst'; length 400-900 $\mu \mathrm{sec}$.
Cycle N:Reference period: Recordings Without Stimulation

Cycle A: Recording with stimulation 1 biphase pulse $2 \times 300 \mu$ s per 1 sec sweep

\section{Resting period}

Cycle B: Recording with stimulation 1 biphase pulse $2 \times 300 \mu$ s per 1 sec sweep

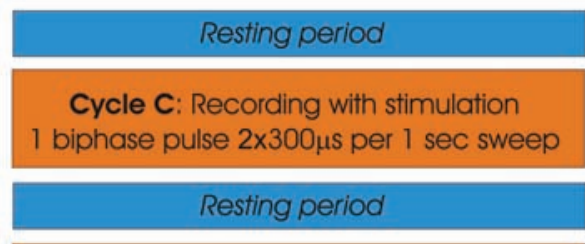

\section{Resting period}

Cycle D: Recording with stimulation biphase pulse $2 \times 300 \mu$ s per 1 sec sweep
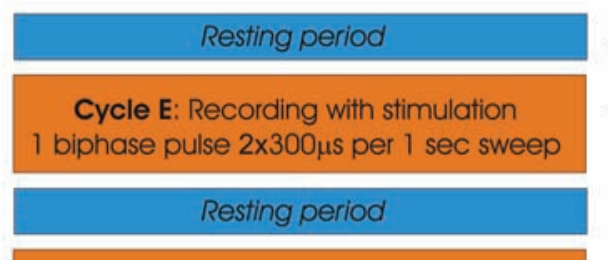

Cycle F: Recording with stimulation 1 biphase pulse $2 \times 300 \mu$ s per 1 sec sweep
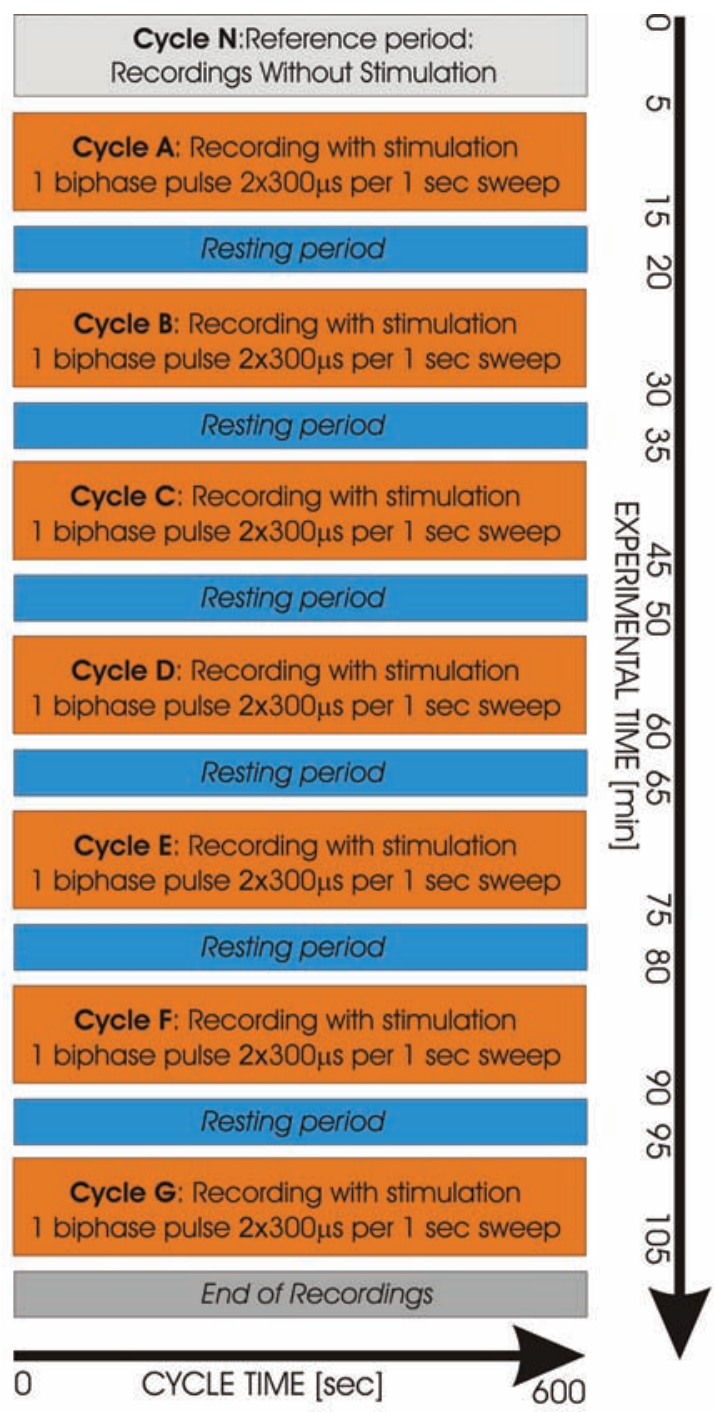

Figure I Flow chart of electrical stimulation. This is an illustration of the stimulusrecording protocol.The protocol starts with recordings for a reference cycle, denoted as N.During this period, no stimulation is applied to the sample and only basal activity is recorded for reference purposes. The experiment is followed by seven cycles, with length of six hundred seconds each. The cycles were denoted with letters A to G. During the cycles, stimuli were applied to the culture as described in the materials and methods (stimulation section). The stimulation cycles were altered with resting cycles for culture recovery for tree hundred seconds for culture recovery.

Many other published definitions are not mentioned because their culture type is incompatible to the one which is the subject of discussion here.

\section{Immunostaining under light microscopy}

Spheroids were sectioned at thicknesses of $5 \mu \mathrm{m}$ and stained with Neurofilament (Clone:2F11, Mouse Monoclonal, Lab Vision Co. UK) with secondary conjugated Alexa Fluor 555 $\mathrm{F}\left(\mathrm{ab}^{\prime}\right) 2$ (Fragment of Goat anti-Mouse, $\operatorname{Ig}(\mathrm{H}+\mathrm{L}) * 2 \mathrm{mg} / \mathrm{ml}$, Molecular Probes) and Glial Fibrilliary Acidic Protein (AB-4, 51-52 kDa, Rabbit Polyclonal AB, Lab Vision Co. UK) with secondary conjugated Alexa Fluor 488 F(ab')2 (Fragment of 
Goat anti-Rabbit, Ig $(\mathrm{H}+\mathrm{L}) * 2 \mathrm{mg} / \mathrm{ml}$, Molecular Probes) for 30 minutes with dilutions of 1:50. The samples were imaged with a confocal laser scanning microscope Nikon PCM2000 equipped with $488 \mathrm{~nm}$ (Argon) and $543 \mathrm{~nm}$ (HeNe) laser lines under Nikon/Coord EZ2000 software (Nicon BV, The Netherlands).

\section{Data analysis and presentation}

The entire data was collected over two and a half months of the lifetime of the spheroids and, as a consequence, a comprehensive set of data of approximately 340 GB was recorded.

\section{Analysis of the spiking frequency}

A brief illustration of the type of analysis is presented in Figure 3. Due to their complexity, a statistical approach is taken to analyze the spiking behavioral differences under various circumstances. An illustration of the spiking frequency in time is presented together with their statistical distributions with fits in Figure 3A. This way, both a qualitative and quantitative estimate of the activity may be undertaken by direct observation of the histogram parameters. We were intrigued as to whether the electrical stimulus is inducing significant changes that may reflect in the overall spiking activity within the cycles A to G. To understand and measure this behavioural change within the stimulating cycles, the difference in the spiking frequency distribution (A vs. B, A vs. C, A vs. D, A vs. E, A vs. F, and A vs. G) was tested with a Kolmogorov-Smirnov (Matlab, MathWorks, CA) statistical test and empirical cumulative distribution (ECDF) plots were produced, as illustrated in Figure 3B. In the resulting ECDF plots, some test parameters are indicated as follows: $h=0$ indicating 'equal' or 1-'different' distribution; $p<0.05$ level on all tests; and a letter 'A' - 'G' which indicates the cycle. The quantitative and qualitative (Figures $3 \mathrm{E}$ and $3 \mathrm{D}$ ) changes in behavior are presented by the change in the overall dynamics indicated with arrows in Figure 3C as 'right shift', 'left shift' and 'no shift' of the spiking frequency distributions found in comparison to Figure $3 \mathrm{D}$ as a quantitative indicator.

\section{Spiking frequency dynamics (A cycles shown) in lifetime}

To show the dynamics of the spiking activity, spiking frequency plots (Only ' $A$ ' periods) are presented across the lifetime of a typical spheroid in Figure 2. Other illustrations of the dynamics of mean spiking frequency ratio during the lifetime of the spheroids are shown in Figure 6. The figure shows a spiking frequency ratio, formed by the relationship between the mean spiking frequency within the stimulated period $A$ (recording with stimulation) and
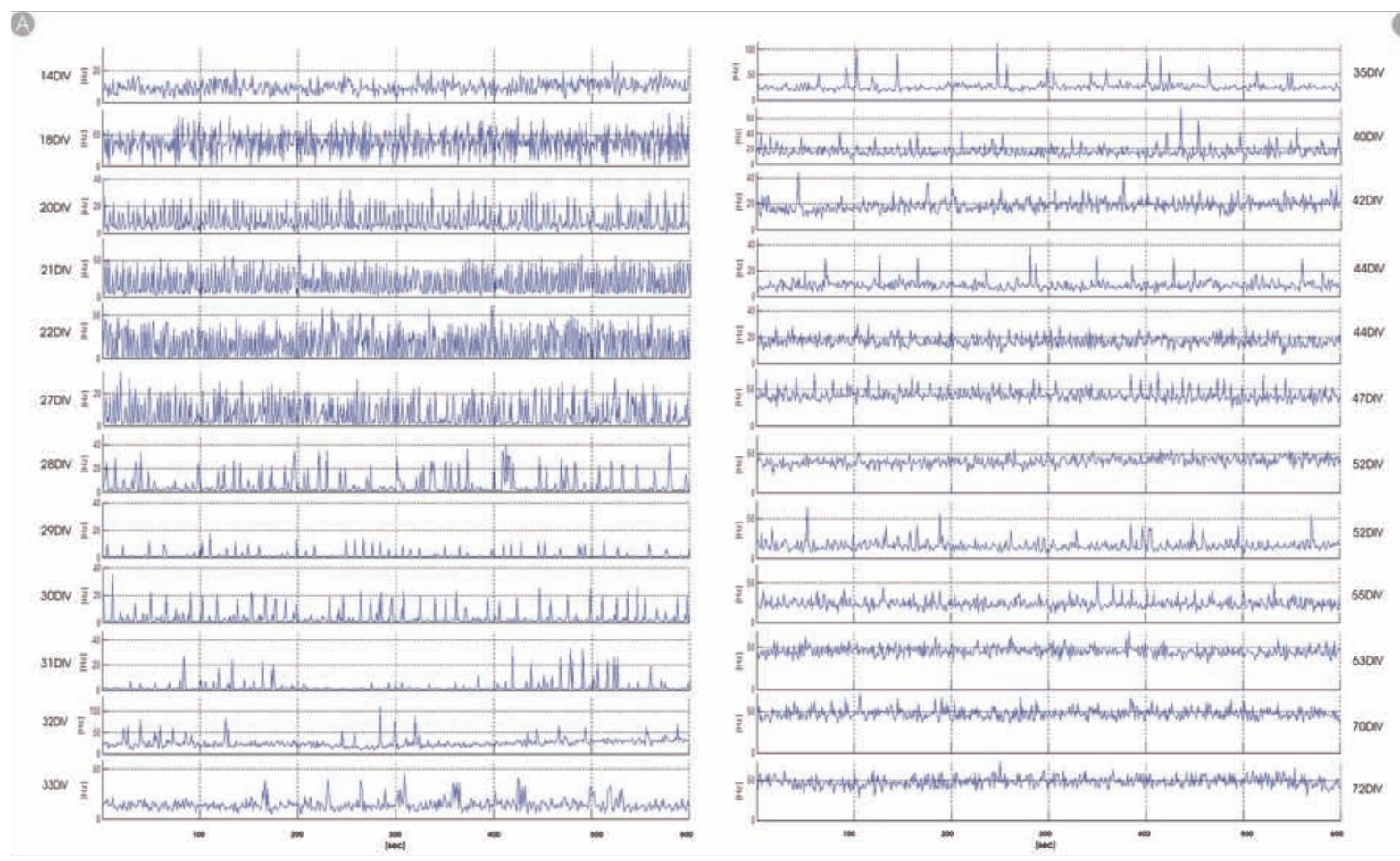

Figure 2 Spiking development. A comprehensive data set of spiking frequency plots, for the first stimulating period ' $A$ ', within the lifetime of the cultures is presented. Two columns of plots denoted as A and B showed typical spiking activity on the frequency plots within the life period between DIV I4 and DIV 72. 

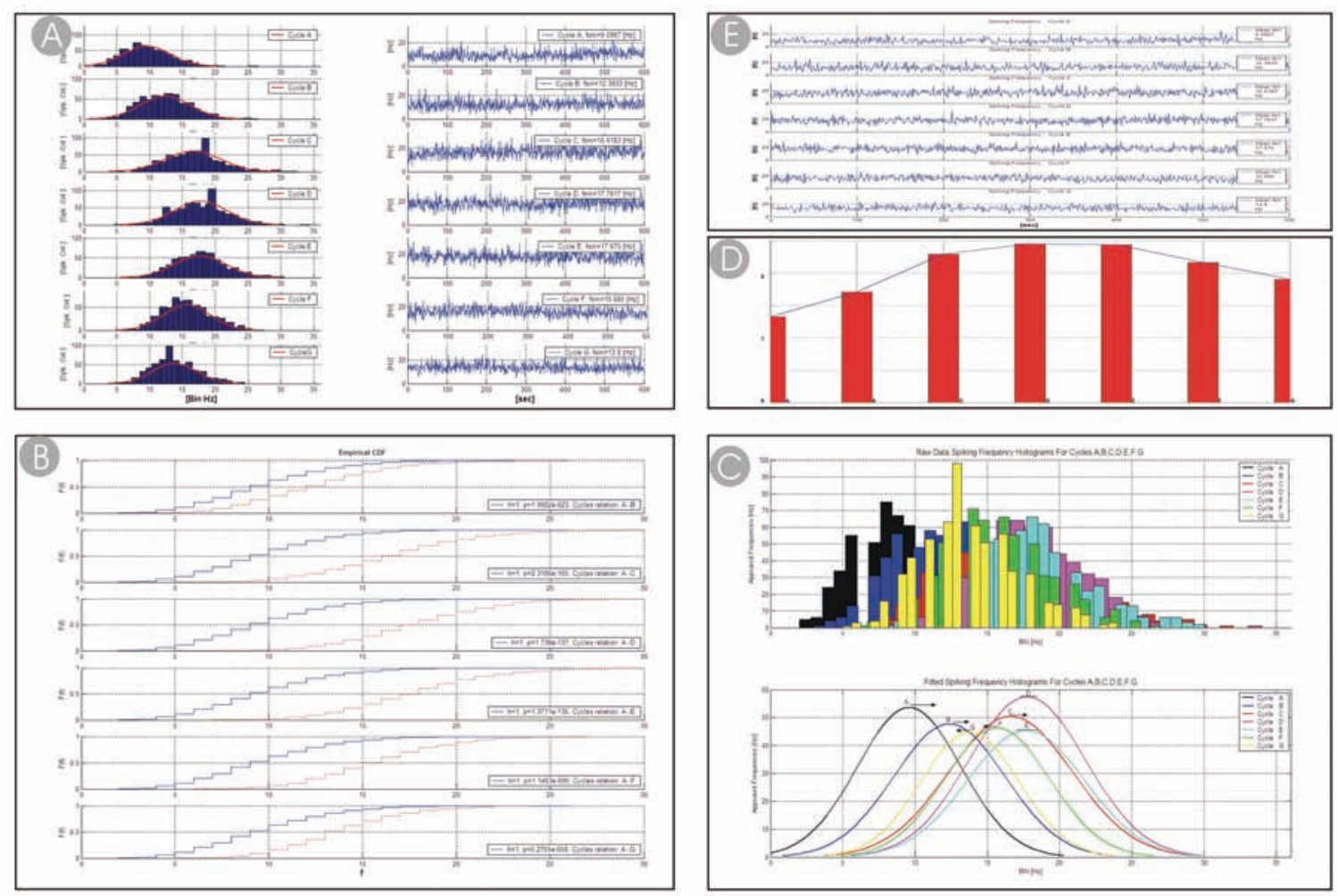

Figure 3 Illustration of data analysis. The data was recalculated for spiking frequency estimation and presented as frequency plots vs. time, together with the spiking frequency distributions and their fits on A.To analyze the difference in the spiking behavior under stimulation between B, C, D, E, F, G cycles and the first stimulated cycle A, a KolmogorovSmirnov (Matlab, MathWorks) test for difference in the spiking frequency distributions (A vs. B,A vs. C,A vs. D,A vs. E,A vs. F,A vs. G) was performed and empirical cumulative distribution plots illustrated it on B. On each plot, the test result is indicated by the parameters $h(0=$ equal, $I=$ different $), p(p-v a l u e, w h e r e ~ p<0.05)$ and cycles relation. To show the dynamics of the ability of the network to respond to stimulus within long term electrical stimulation, the spiking frequency ratio was estimated as the mean spiking frequency under stimulation, for each recording cycle (A to $G$ ), normalized to the mean spontaneous spiking frequency (cycle $N$ ). This way, $\mathbf{D}$ shows, in a quantitative way, the spiking frequency ratio of data from DIV 14 (cycles $A$ to $G$ ) sample where $E$ shows the corresponding spiking frequency plots to the cycles $A$ to $G$. To unveil the dynamics of the response to the stimuli in time, a plot is showing the raw data histograms and their fits on $\mathbf{C}$. The shift from the left to right (cycles $\mathrm{A}, \mathrm{B}, \mathrm{C}$ marked with right arrows) is showing the increase of the activity, (cycles D, E marked with lines) where a dynamic hold is observed and right to left (cycles F, G marked with left arrows) where a decrease in the frequency was found. In this case we highlight on 3 periods, where the frequency ratio rises, stay flat, and falls down.

the reference period $N$ (recording without stimulation). This is a quantitative estimate of the averaged response of the spheroids to the electrical stimuli. The ratio reflects the effect between a basal period without stimulation and the one at bias condition.

\section{Exhaustion}

To show the effects of exhaustion, where two types of spiking activity decrease were seen, the frequency plots over the stimulation cycles $A$ to $G$ (one particular network) were plotted in Figure $4 \mathrm{~A}$ vs. their levels of the mean spiking frequency ratio Figure 4B in respect to the reference period $N$ on Figure 4C. The mean levels of the spiking frequency ratio were elevated (A and $\mathrm{B}$ periods) and then decreased ( $\mathrm{C}$ to $\mathrm{G}$ ) below the reference period $\mathrm{N}$. This is an exhaustion that reflects the overall activity of the network where the sporadic and train spiking decreased. In the case of prevailing train spiking, exhaustion is observed by the disappearance of the train activity as shown in Figure 5. Both processes may vary with respect to the time measured in cycles A to $G$, but similar patterns of exhaustion were seen in the whole set of samples.

\section{Reaction to the stimuli measured via mean spiking frequency ratio}

The spiking frequency ratio, denoted $f_{s t} / f_{s p}$, shows the relation between the mean spontaneous network activity and the stimulated one. The ratio is one that signifies in an absolute way, since it has no measurement units, the ability of the network to respond to the electrical stimuli; both basal and biased conditions are considered:

$$
f_{s t} / f_{s p}=\frac{\bar{f}_{\text {stimulated }}}{\bar{f}_{\text {spontaneous }}} \text { [absolute number] }
$$

In this way the network is understood as a 'black box processing unit', where an appropriate input (stimulus) is 


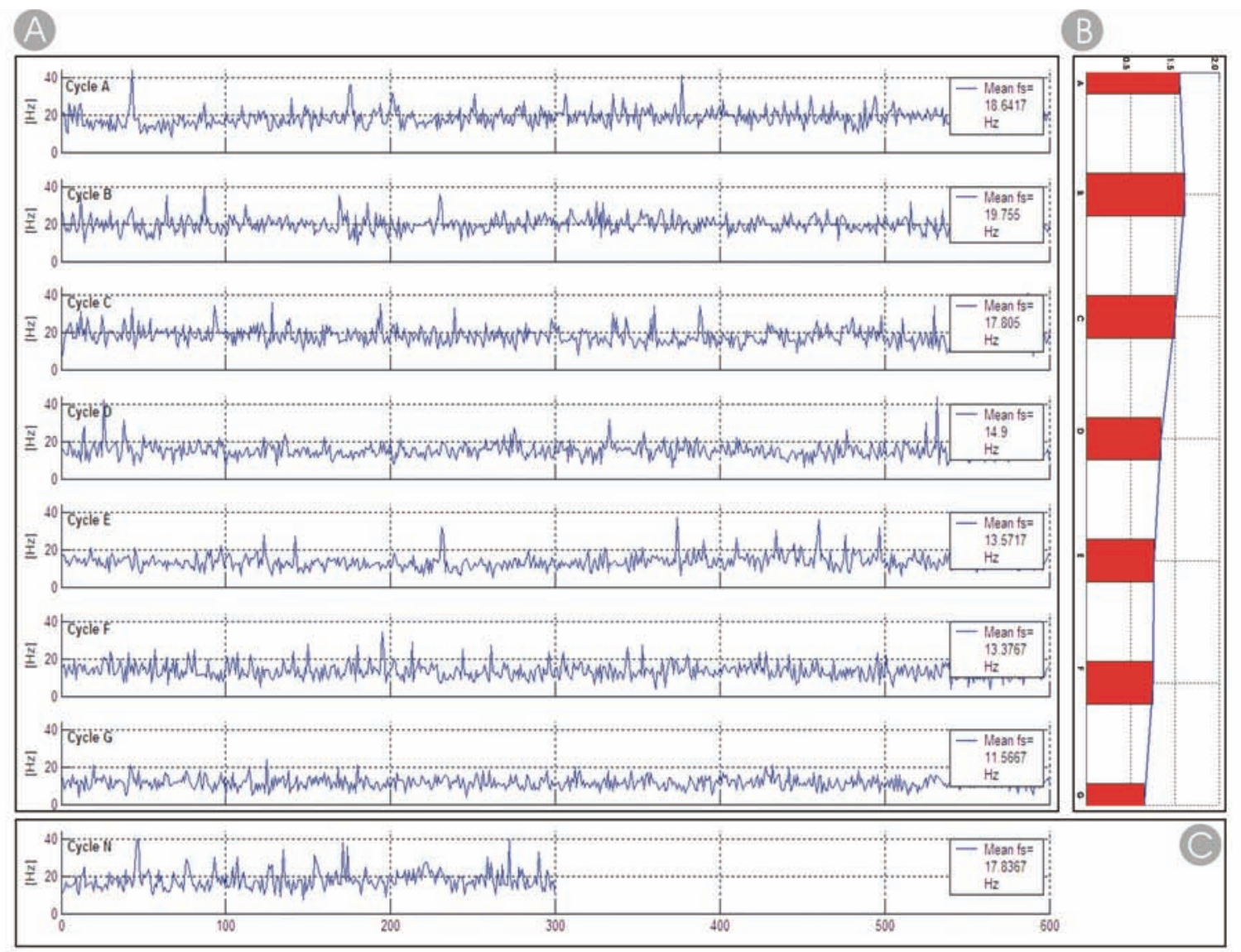

Figure 4 Spiking exhaustion. To show the effect on exhaustion where two types of spiking activity were decreased due to stimuli application, the frequency plots over the stimulation cycles $\mathrm{A}$ to $\mathrm{G}$ were plotted on $\mathbf{A}$ in comparison to $f_{s t} / f_{s p}$ on $\mathbf{B}$, where more quantitatively those changes are observed, in respect to the reference period $\mathbf{N}$ plotted on $\mathbf{C}$.

given and output (recorded output) is analyzed. To illustrate the excitation dynamics of the network caused by stimuli, within the experimental cycles A to $G$, a typical network is shown and traced within its lifetime in Figure 7. The most significant change in the mean frequency ratio is observed between 31 and 35 DIV where the ratio rises from 1.2 up to 6.8 in the time period for the cycles A to G, in the particular example.

\section{Results}

In the following, descriptions of the major features in the spiking behavior at various stages of development are presented. As noted above, the preparation of the cell cultures results in the production of thousands of spheroids. Twenty cell cultures, each on individual MEAs, were chosen for study, primarily based upon their apparent degree of connection to the electrode array surface. The temporal pattern of development remained almost identical in all such samples but a degree of variance in the exact days for a given behavior was experienced. The following groupings of development age represent typical time points.

\section{Stage 14-17 DIV}

Around this stage of maturation, the prevailing spiking behavior is characterized as weak and sporadic, consisting of stochastically arriving spikes termed as sporadic activity or weak CB (continue burst) type. The activity found at this stage for the vast majority is sporadic although some weak trains were recorded toward the end of this lifetime period. Hence the histograms are well defined and Gaussian-like in shape (an example is shown in Figure 3A). Two-peak histograms may be found toward the end of this period; one from the base line of sporadic activity and one from the weak bursting activity caused by trains. The major development in behavior is the transition of spiking from sporadic spikes to organized trains. The baseline (mean spiking activity) is typically around $20-25 \mathrm{~Hz}$ (Figure 3E). The levels of spiking frequency are persistent and may be seen as stable in the frame of the stimulating cycles. There is no significant 

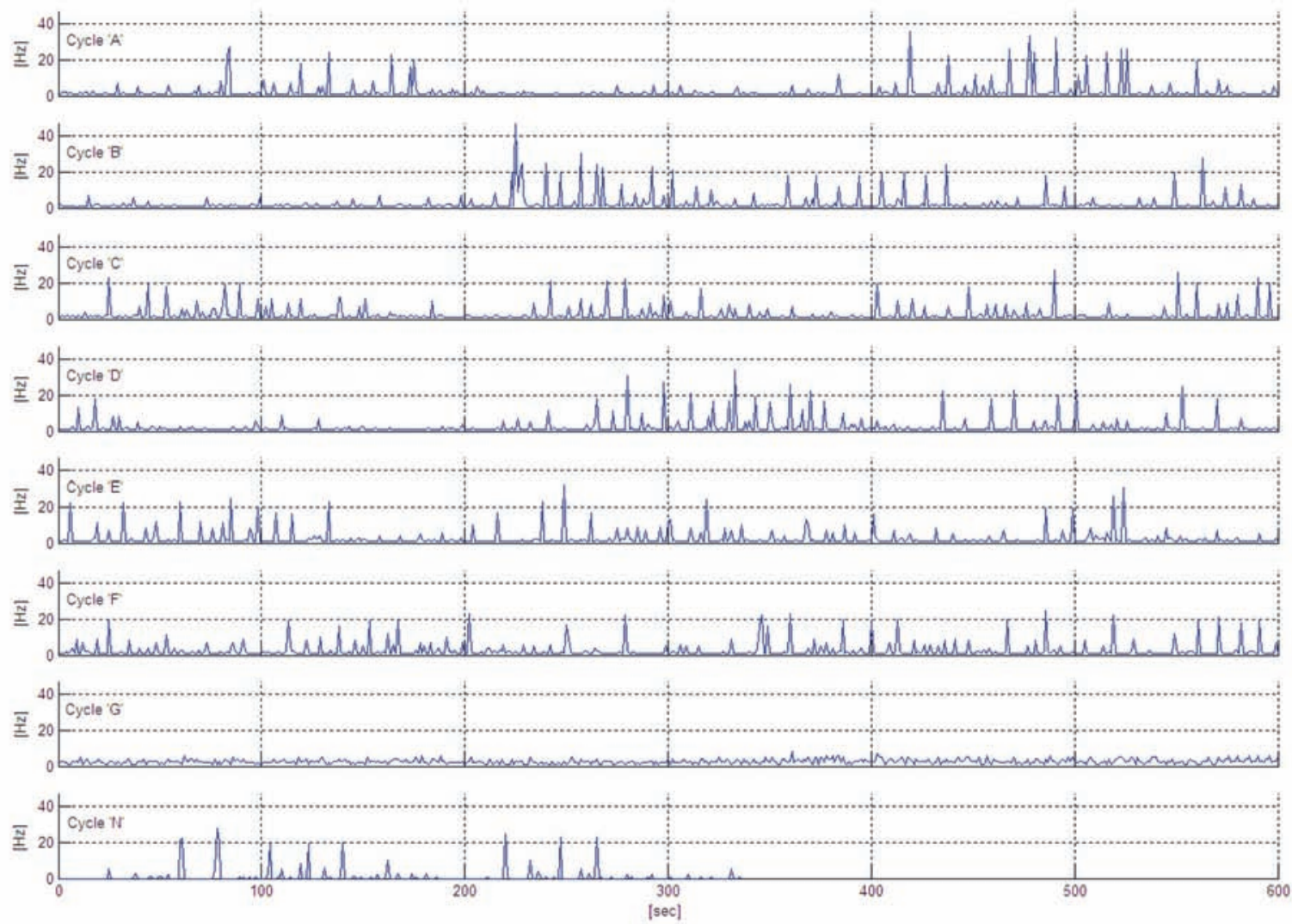

Figure 5 Spiking exhaustion on train activity. Exhaustion is demonstrated toward the last stimulation cycles, ie, G, in the experiment, where the sample is showing clear train spiking which disappeared during the cycle.

exhaustion through the whole experiment. The initial values at the cycle $A$ are typically relatively lower than the following $B, C$ and so on, from which we may conclude an excitatory network reaction from the structure. The ramping of the $f_{s t} / f_{s p}$ is characterized by the gradated slope and a maximum around $E$ may be found as a starting point of weak exhaustion (Figure 3D). The effect of the stimulus application is statistically significant (Figure 3B) and complies with the already reported doubling in the spiking frequency under stimulation (Figure 6). The small variation in network development during this period means most spheroids reach a doubling of spiking frequency in the early cycles of stimulation but some others are slightly later, although all clearly show this effect from the stimulation. The histogram shifts are typically to the right at this stage because of the ramping up the ration of $f_{s t} / f_{s p}$ (Figure 3C).

\section{Stage |8-2I DIV}

In the beginning (18-19 DIV) the stage is presented by a mixture of $\mathrm{CB}$, which is characterizing the level of sporadic behavior, and MDB (massive dense bursts) that gives an organization into trains. Some samples are able to demonstrate
MDBI (MDB intervals) activity. Others show a mixture, ie, MDBI together with some sporadic background CB activity. This is an indicator for the developing stage that may be related to tightening the trains and reducing the sporadic nature in order to become more controllable and inhibiting the spontaneous response. Toward the end of this period, distinguishable trains appeared with high frequency; approximately one every $2-3$ sweeps $(0.5-0.3 \mathrm{~Hz})$. The difference between train frequencies $(\sim 30-40 \mathrm{~Hz})$ and the base line $(\sim 5-8 \mathrm{~Hz})$ is a significant and clear indicator for the mixed type of behavior observed. The mean spiking frequency was persistent during the stimulating cycles, although the spiking frequency within the cycles (A to $G$ ) decays, which indicates an exhaustion due to the stimulation process (Figure 4A). The comparison between stimulated and nonstimulated frequency plots shows an elevation of the train activity expressed in long peaks for the trains (an example is shown in Figure 2). At this stage, we observed more samples where the $f_{s t} / f_{s p}$ has a downwards slope which indicates a tendency for exhaustion due to the stimulus application in time (Figure 4B). The reasonable distance between spontaneous and stimulated mean values of the spiking frequency is a sign of a strengthening in network 


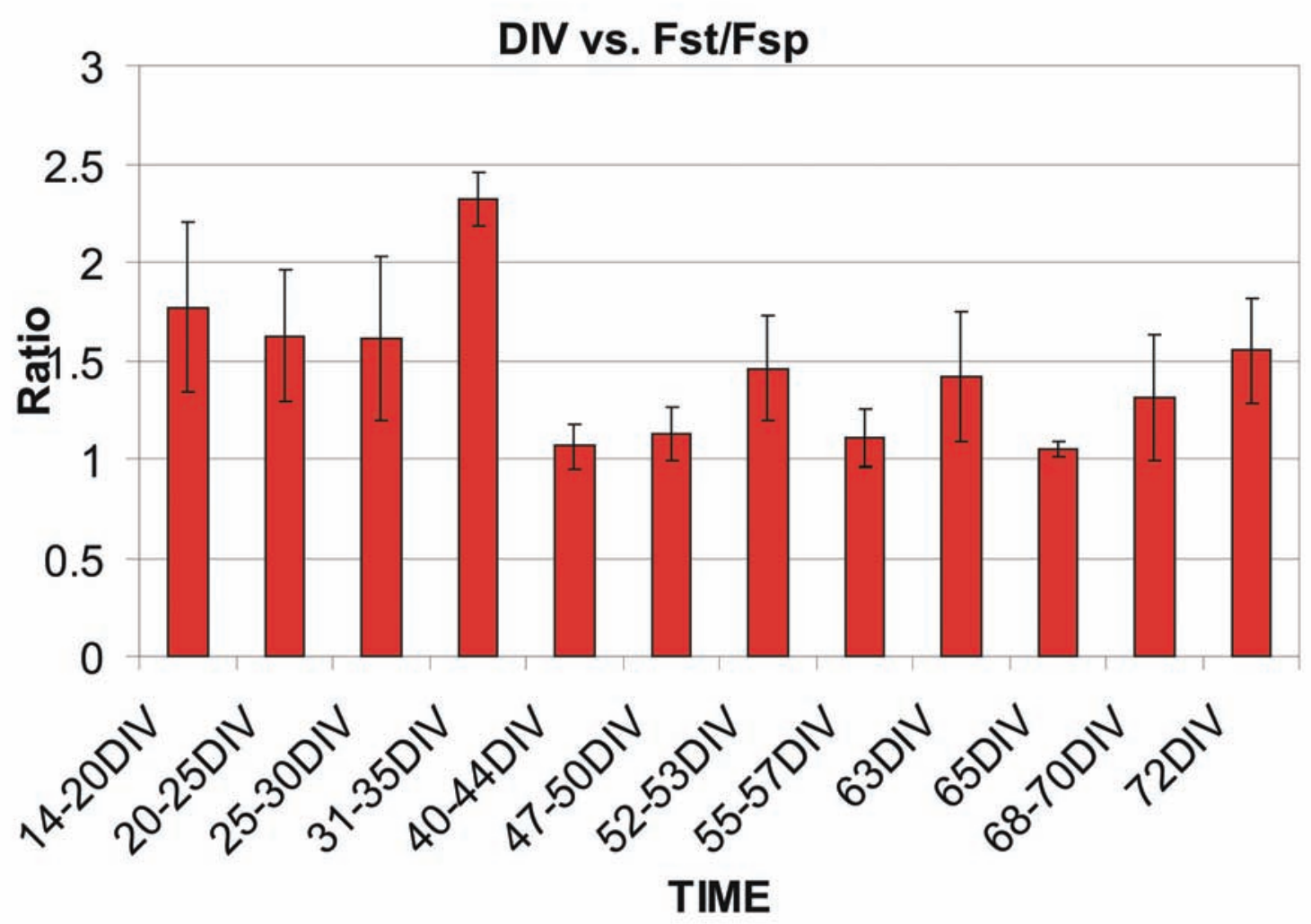

Figure 6 Ratio of stimulated and spontaneous spiking frequencies. The frequency ratios $f_{s t} / f_{s p}$ for cycle A, between stimulated and spontaneous activity, across the lifetime of the cultures is shown.

connectivity. The distributions are typically two peaks, one associated with the baseline of $\mathrm{CB}$ activity and the other with the mean train frequency.

\section{Stage 22-24 DIV}

MDBI activity was found in well pronounced trains, with a frequency of $25-35 \mathrm{~Hz}$ rarely approaching $40-50 \mathrm{~Hz}$, and with a weak presence of sporadic activity, ie, CB. The train appearance is at approximately $0.5 \mathrm{~Hz}$ which then decreases over the period to approximately $0.25 \mathrm{~Hz}$ (Figure 2). This is a sign of changes in the network state. The typical number of spikes per train remains relatively unchanged however; comparison between the spontaneous and stimulated spiking frequencies shows no change over the cycles $A$ to $G$ (Figure 7). The histogram of spiking frequencies is twin-peaked, referring to minor sporadic spikes and major train activity.

\section{Stage 25-27 DIV}

Toward the end of this period, an absence of sporadic activity was found which indicates the self inhibition of the spontaneous behavior. The adaptation of the network to the stimulus is observable later in cycles $A$ to $G$ with an increase in the value of the $f_{s t} / f_{s p}$ of up to around 1.5 on average (an example is shown in Figure 7). This shows strong network organization caused by the maturation process. In some cases there is a decline around cycles $\mathrm{F}$ and $\mathrm{G}$. The histograms tend to be two - more separated - peaks than in the previous period, ie, there is only a minor sporadic $\mathrm{CB}$ component to the activity (Figure 2). This pattern remains until around $31 \mathrm{DIV}$. The spiking exhaustion is observed on two levels, one associate with train disappearance and another with possible elevation of the sporadic activity within A-G cycles. The exhaustion patterns tend to repeats with better pronunciation toward the 46 DIV.

\section{Stage 28 DIV}

Spiking in MDBI and MDB was recorded as sparsely spaced spike trains coming approximately every one in ten seconds. They are characterized by a high spike frequency in comparison to the mute periods between them (Figure 2). With the stimulus cycles A to G, a grouping of trains is observed and train appearance is about 7-15 trains per 100 seconds which is a sign of network behavior development due to the stimulation process. The levels of spiking frequencies are persistent and remain stable till the end of the experiment. The total activity is classified 

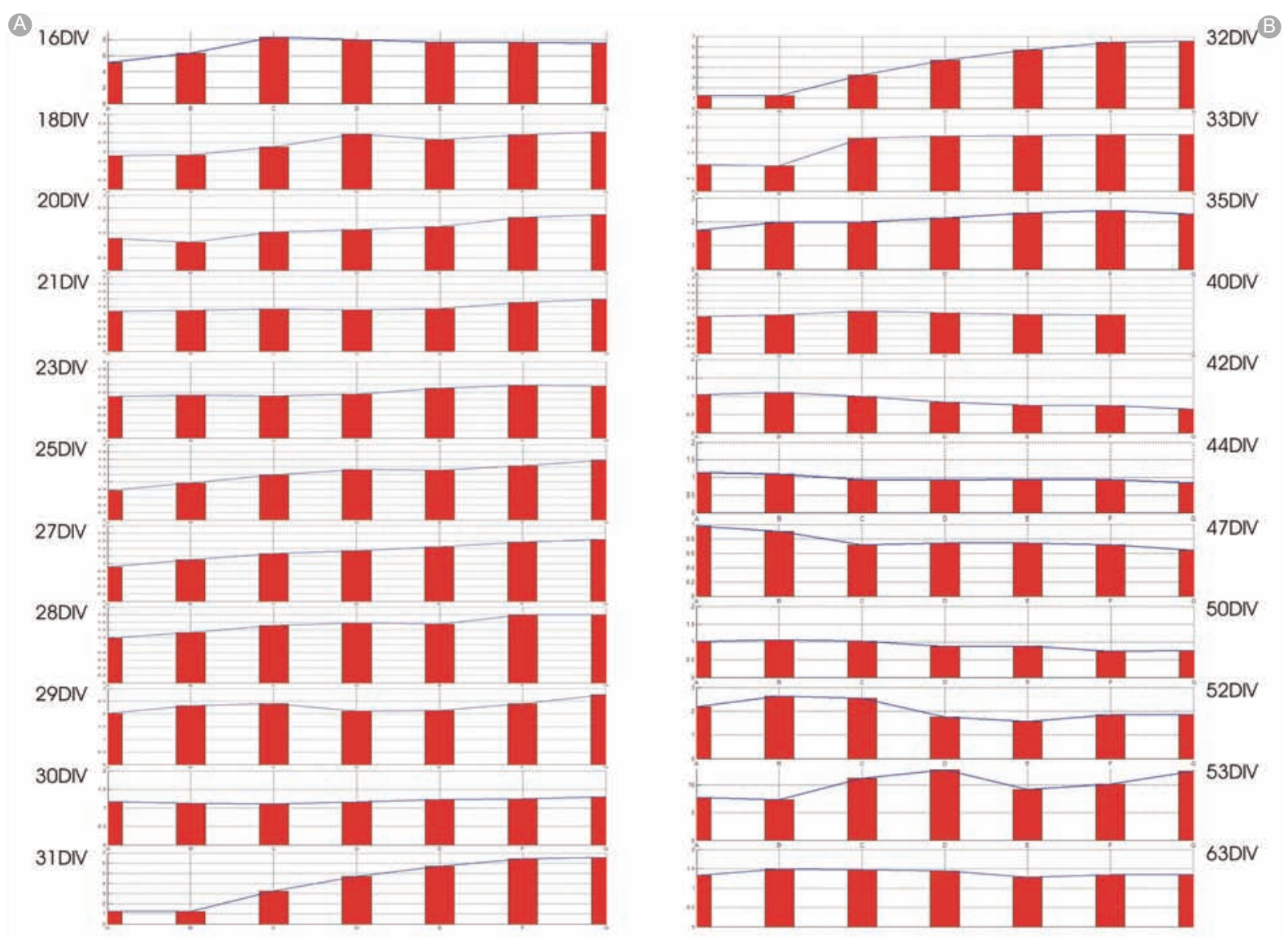

Figure $7 \mathrm{~A}-\mathrm{G} f_{s t} / f_{s p}$ dynamics for particular network in 16-63 DIV. The spiking frequency ratio $f_{s t} / f_{s p}$ of network, shows the dynamic of the $A$ to $G$ cycles with in the culture lifetime DIV 16-DIV 63.

as strong because the mean values are above $30 \mathrm{~Hz}$, some samples even $100 \mathrm{~Hz}$. The $f_{s t} / f_{s p}$ ratio posses up and down slopes within $A-G$ cycle. This reaction to the stimuli indicates an adaptation followed by possible flat ratio and exhaustion by the end of $\mathrm{A}-\mathrm{G}$ cycles. The exhaustion was observed on spike-trains and sporadic activity change within $A-G$ cycles, where similar patterns were observed till 30 DIV.

\section{Stage 29-30 DIV}

This stage is classified as well organized trains where an average level of the trains is slightly decreased toward 30 DIV and a greatly reduced amount of $\mathrm{CB}$ behavior is observed (Figure 2). The presence of relatively high sporadic mean levels in comparison to train appearance is a sign of a significant transformation in network behavior. The frequency measures were persistent within the stimulation cycles $\mathrm{A}-\mathrm{G}$.

\section{Stage 3I-35 DIV}

Here a very weak sporadic baseline activity is seen and strongly grouped trains in large formations (MDB/MDBI) are dominant initially in the period. Increasing amounts of CB behavior is seen, typically up to around $20 \mathrm{~Hz}$, increasing further to around $45 \mathrm{~Hz}$ within the stimulating cycles A-G. Under the stimulation cycles MDBI activity increases significantly or more typically, decreases. MDBI can contain spikes at around $200 \mathrm{~Hz}$ per train but the frequency of their arrival decreases or disappears due to exhaustion (Figure 5). The overall increased frequency ratio within A-G (Figure 7) is very marked which suggests the network is able to produce maximally elevated levels of excitation in response to the stimulus. The two clear peaks in the histograms of spiking activity start to become increasingly merged back into a single Gaussian distribution around DIV72.

\section{Stage 36-46 DIV}

The recordings showed a relatively low but persistent level of sporadic (CB) activity around $15 \mathrm{~Hz}$ and well shaped frequency peaks indicating the train burst activity reaching values $50-60 \mathrm{~Hz}$. The persistency in the sporadic activity under the cycles A-G is a sign for a nucleus in the network, where stable activity is generated and retained till the end 

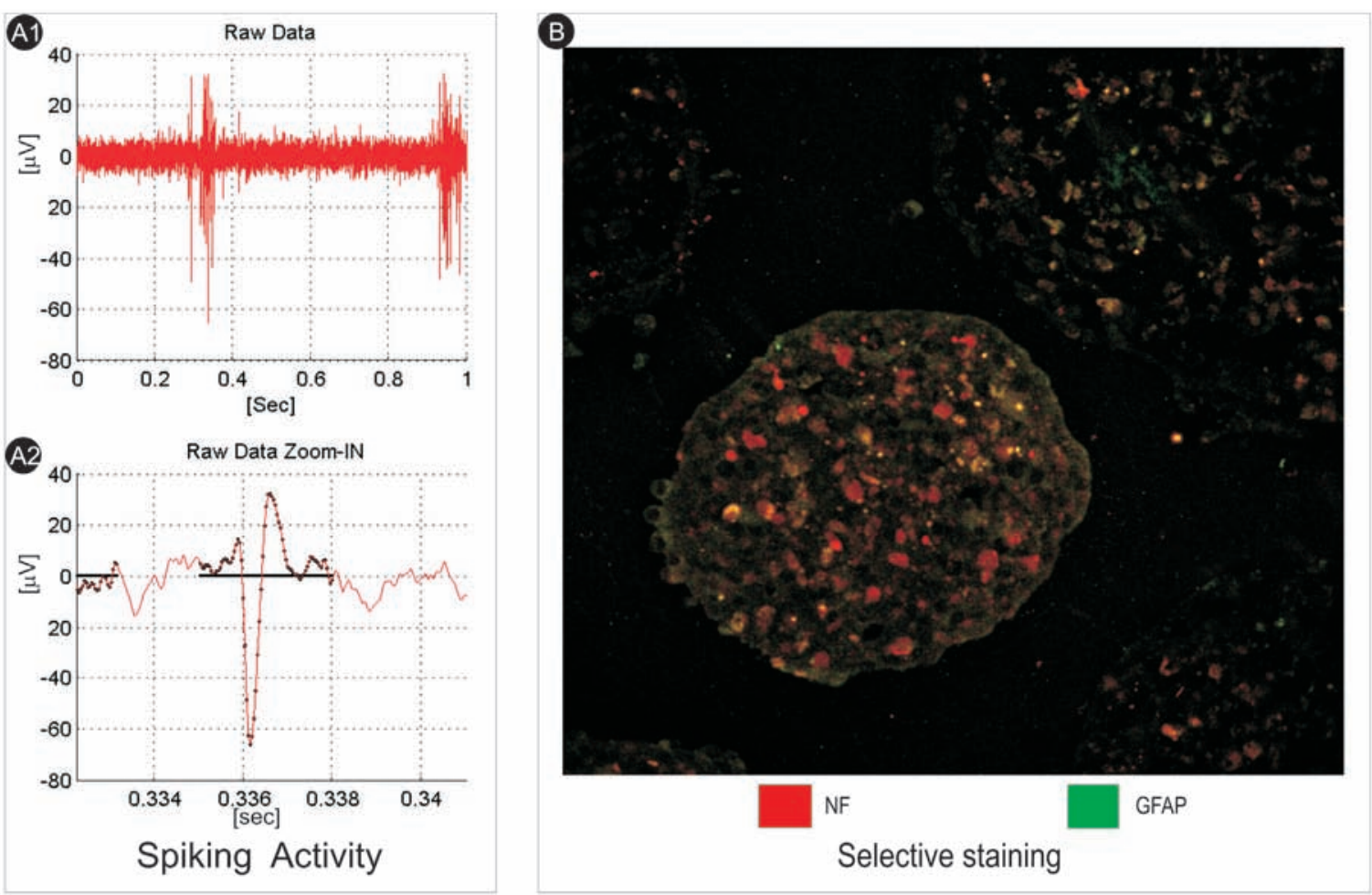

Figure 8 GFAP/NF staining. AI.A raw data signal is shown, one on a I sec time scale, were two spike trains are depicted. A2. The time scale was stretched out to I0 msec scale $(0.332$ to $0.338 \mathrm{sec})$, to be able to show a single spike. The approximate spike time is in the range of I.5 ms. B. GFAP/NF staining of thin spheroid's section. GFAP is largely covering the sections where glia cells are indicated by green and centralized neuronal distribution, NF stain, is marked by red.

of the experiment. The change in activity is not significant under the cycles A-G compared with the spontaneous activity, ie, $f_{s t} / f_{s p}$ is around 1 .

\section{Stage 47-52 DIV}

In the present stage, the stable sporadic activity is observed in all the recorded networks with persistent levels till the end of the cycles. Some train activity is emerging in an irregular manner. The train frequency is considered to be the similar to the previous period (36-46 DIV). Under the stimulation cycles $A-G$, the frequency ratio tends to fade indicating exhaustion in the level of sporadic activity.

\section{Stage 53-72 DIV}

Over these later stages of development a high level of CB activity is observed and almost no train activity signifying another significant stage in network development. Over the stimulation cycles $\mathrm{A}-\mathrm{G}$ the ratio $f_{s t} / f_{s p}$ doesn't show a clear tendency but is sometimes around 1 up to around 1.5.

\section{Neurofilamen and glial fibrillary acidic protein staining of spheroids sections}

Thin slices were produced and stained for NF (neuro filament) and GFAP (glial fibrilliary acidic protein) and are shown in Figure 8. The GFAP is marked with green which can be seen to be widely spread over the entire section. The NF is presented nearly in the centralized area of the section and imaged with red where cells are.

\section{Discussion}

Figure 6 demonstrates the overall effect of stimulation with respect to the spontaneous activity of the network within the spheroids' lifetime. As may be seen from the data, the majority of the samples at the beginning showed an increased ability to respond to the stimuli as we previously reported (Uroukov et al 2006) wherein the frequency ratio is in the range of $1.8-2 \mathrm{~Hz}$. Within the next $10 \mathrm{DIV}$, this ratio gradually decreased to $1.6-1.7 \mathrm{~Hz}$. In this period, the spiking was a mixture comprised from some sporadic activity, characterized by a decrease in the stochastic component, and a transfer to patterned spiking structures of train formations. Those formations varied in length and were changing according to the DIV. Figure 2 is able to demonstrate the persistence of the train sizes during the experiments where at 14-18 DIV they vary and hide within the dominant sporadic activity. As a result of the subsequently reduced sporadic activity, more organized and persistent trains were observed at 22-27 DIV. 
At the stage 28-31 DIV, the activity undergoes a major spiking behavioral change and trains become very much the prevailing behavior. At 31 DIV a grouping of the train formation was observed in time where mute periods were also found. The total activity was organized in massive train formations grouped in large train clusters. This significant change may be due to the innate network maturation as it may be associated with the growing of the animal in vivo that is similar to the hatching cycle of chicks where the chicks emerge with developed motor functions. In a similar time span, the reactivity of the network was incredibly strong to the stimuli in comparison to the vast majority of cases. This may be seen through the raised frequency ratio which is illustrated in Figure 7. Meantime, on the spiking organization scale, some sporadic activity was found to rise and in general increased until the end of the lifetime. The mean levels may vary but the activity presence is confirmed. Some interesting close proximity subclustering was observed around 32-33 DIV where the sporadic activity was found to increase. A very interesting prominent activity between 35 DIV and 40 DIV emerged as stochastic strong and tight trains. Because of the stochasticity, the frequency ratios were found to be very low in comparison to 30-35 DIV. Towards the end of the lifetime, after 55 DIV, the stochastically arriving unorganized spikes were the most prevalent and the activity may be described as one without inhibition. However we do not consider this stage as epileptic as described in (Wagenaar et al 2005) because of the dynamics that may still be seen at later DIV in Figure 7.

\section{Conclusion}

In this article we have performed a long-term stimulation study of hen embryo brain spheroids to establish the baselines for long-term stimulation experiments. The effect on spiking exhaustion was described in terms of the culture lifetime. The change in the spiking frequency with respect to the spike organization was analyzed and associated to spiking exhaustion in the cell culture in experimental and lifetime scales. Concerning the cell culture development, two main types of spiking exhaustion were described, taking into account the stimuli on a long experimental scale: one which exhibits a drop of the sporadic spiking frequency at the latest experimental stages and another associated with decreasing spike train appearance. The most sensible way is to envisage the culture response to stimuli and activity through development lifetime. We found two major characteristics to reach their optimum at similar lifetime periods of the cultures: the spiking frequency ratio and the spiking train organization. We conclude 14-35 DIV is the most suitable for future plasticity experimentation.

\section{Disclosure}

ISU designed this particular study. ISU has performed the cell culturing, data acquisition, data analysis and prepared this manuscript. LB designed and controlled the main framework of the project and contributed to the manuscript preparation. This work was supported under EPSRC grant no. GR/ T11029/01. The authors report no conflicts of interest in this work.

\section{References}

Babb TL, Soper HV, Lieb JP, et al. 1977. Electrophysiological studies of long-term electrical stimulation of the cerebellum in monkeys. J Neurosurg, 47:353-65.

Bull L, Uroukov I. 2007. Initial results from the use of learning classifier systems to control in vitro neuronal networks. In: Lipson $\mathrm{H}$, et al. (eds). GECOO-2007: Proceedings of the Genetic and Evolutionary Computation Conference. ACM Press.

Darbon P, Scicluna L, Tscherter A, et al. 2002. Mechanisms controlling bursting activity induced by disinhibition in spinal cord networks. Eur J Neurosci, 15:671-83.

DeLong GR. 1970. Histogenesis of fetal mouse isocortex and hippo-campus in reaggregating cell cultures. Dev Biol, 22:563-83.

Demarse TB, Wagenaar DA, Blau A, et al. 2001. The neurally controlled animat: biological brains acting with simulated bodies. Autonomous Robots, 11:305-10.

Eytan D, Brenner N, Marom S. 2003. Selective adaptation in networks of cortical neurons. J Neurosci, 23:9349-56.

Goldsmith PC, Berens ME. 1990. Methods in Neuroscience. London: Academic Press, Vol 2, pp. 236-64.

Gross GW. 1979. Simultaneous single unit recording in vitro with a photoetched laser deinsulated gold multimicroelectrode surface. IEEE Trans Biomed Eng, 26:273-9.

Guillory KS, Norman RA. 1999. A 100-channel system for real time detection and storage of extracellular spike waveforms. $J$ Neurosci Methods, 91:21-9.

Güven D, Weiland JD, Fujii G, et al. 2005. Long-term stimulation by active epiretinal implants in normal and RCD1 dogs. J Neural Eng, 2:65-73.

Hamburger V, Hamilton H. 1951. A series of normal stages in the development of the chick embryo. J Morphol, 88:49-92.

Heuschkel MO, Fejtl M, Raggenbass M, et al. 2002. A threedimensional multi-electrode array for multi-site stimulation and recording in acute brain slices. J Neurosci Methods, 114:135-48.

Jimbo Y, Kawana A, Parodi P, et al. 2000. The dynamics of a neuronal culture of dissociated cortical neurons of neonatal rats. Biol Cybern, 83:1-20.

Jimbo Y, Robinson HPC, Kawana A. 1998. Strengthening of synchronized activity by tetanic stimulation in cortical cultures: Application of planar electrode arrays. IEEE Trans Biomed Eng, 45:1297-304.

Jimbo Y, Tateno T, Robinson HPC. 1999. Simultaneous induction of pathwayspecific potentiation and depression in networks of cortical neurons. Biophys $J, 76: 670-8$.

Morefield SI, Keefer EW, Chapman KD, et al. 2000. Drug evaluations using neuronal networks cultured on microelectrode arrays. Biosens Bioelectron, 15(7-8):383-96.

Pine J. 1980. Recording action potentials in cultured neuronal networks with extracellular microcircuit electrodes. J Neurosci Methods, 2:19-31. 
Ruaro ME, Bonifazi P, Torre V. 2005. Toward the neurocomputer: Image processing and pattern recognition with neuronal cultures. IEEE Trans Biomed Eng, 52:371-83.

Sales KM, Kingston ST, Doyle KM, et al. 2004. Preliminary characterisation of an in vitro paradigm for the study of the delayed effects of organophosphorus compounds: hen embryo brain spheroids. Toxicology, 195:187-202.

Seeds NW. 1971. Biochemical differentiation in reaggregating brain cell culture. Proc Nat Acad Sci USA, 68:1858-61.

Shahaf G, Marom S. 2001. Learning in networks of cortical neurons, J Neurosci, 21:8782-8.

Shu Y, Hasentaub A, Azzazy H, et al. 2003. Turning on and off recurrent balanced cortical activity. Nature, 423:288-93.

Stett A, Eggert U, Guenther E, et al. 2003. Biological applications of microelectrode arrays in drug discovery and basic research. Anal Bioanal Chem, 377:486-95.

Tateno T, Jimbo Y, Robinson HPC. 2005. Spatio-temporal cholinergic modulation in cultured networks of rat cortical neurons: evoked activity. Neuroscience, 134:439-48.
Thomas CA, Springer PA, Loeb GE, et al. 1972. A miniature microelectrode array to monitor the bioelectric activity of cultured cells. Exp Cell Res, 74:61-6.

Uroukov IS, Ma M, Bull L, et al. 2006. Electrophysiological measurements in three-dimensional in vivo-mimetic organotypic cell cultures: Preliminary studies with hen embryo brain spheroids, Neurosci Lett, 404:33-8.

Wagenaar DA, Madhavan R, Pine J, et al. 2005. Controlling bursting in cortical cultures with closed-loop multi-electrode stimulation. J Neurosci, 25:680-8.

Wagenaar DA, Pine J, Potter SM. 2004. Effective parameters for stimulation of associated cultures using multi-electrode arrays. J Neurosci Methods, 138:27-37.

Wagenaar DA, Pine J, Potter J. 2006. An extremely rich repertoire of bursting patterns during the development of cortical cultures, $B M C$ Neurosci, 7:11. 